\title{
Dissipative dynamics within stochastic mean-field approach
}

\author{
Ibrahim ULGEN ${ }^{\mathrm{a}, \mathrm{b}, *}$, Bulent YILMAZ ${ }^{\mathrm{a}}$ \\ a Ankara University, Faculty of Sciences, Physics Department, 06100 Ankara, Turkey \\ b Siirt University, Faculty of Sciences and Arts, Physics Department, 56100 Siirt, Turkey
}

\begin{tabular}{l} 
A R T I C L E I N F O \\
\hline Article history: \\
Received 15 October 2019 \\
Received in revised form 10 December 2019 \\
Accepted 10 December 2019 \\
\hline Keywords: \\
Mean-Field \\
Stochastic Mean-Field \\
Fluctuation \\
Dissipation \\
Fermi-Hubbard Model
\end{tabular}

\begin{abstract}
A B S T R A C T
The time-dependent Hartree-Fock (TDHF) and density functional theory (DFT) are among the most useful approaches within mean-field theories for studying static and dynamic properties of complex many-body systems in different branches of physics. Despite the fact that they provide a good approximation for the average properties of one-body degrees of freedom, they are known to fail to include quantal fluctuations of collective observables and they do not provide sufficient dissipation of collective motion. In order to incorporate these missing effects the stochastic mean-field (SMF) approach was proposed (Ayik 2008). In the SMF approach a set of stochastic initial one-body densities are evolved. Each stochastic one-body density matrix consists of a set of stochastic Gaussian random numbers that satisfy the first and second moments of collective one-body observables. Recent works indicate that the SMF approach provides a good description of the dynamics of the nuclear systems (Yilmaz et al. 2018; Ayik et al. 2019). In this work, the one-dimensional Fermi-Hubbard model is simulated with the SMF approach by using different distributions such as Gaussian, uniform, bimodal and two-point distributions. The dissipative dynamics are discussed and the predictive power of the SMF approach with different probability distributions are compared with each other and the exact dynamics. As a result it is shown that by considering different distributions, the predictive power of the SMF approach can be improved.
\end{abstract}

(C) 2019. Turkish Journal Park Academic. All rights reserved.

\section{Introduction}

Mean-field approach is practical tool for studying complex many-body systems. The complexity comes from the number of degrees of freedom (DOF) to be taken account. Increasing number of the DOFs prohibits computation of the exact evolution of the system. This difficulty necessitates to perform simplifications where much less DOFs are considered. One of them which is extensively used is to assume a hierarchy between degrees of freedom according to their complexities. The coupled differential equations which are known as Bogoliubov-Born-Green-Kirkwood-Yvon (BBGKY) hierarchy of equations give the dynamical evolution of different order of densities (one-body, two-body, etc.) (Bogoliubov 1946, Born and Green 1946, Kirwood 1946). The truncation of the BBGKY equations at first level and neglecting all order of correlations give the mean-field equation for the dynamics of one-body density (Lacroix and Ayik 2014)

$i \hbar \frac{\partial \rho}{\partial t}=[h[\rho], \rho]$ $h[\rho]$ is the mean-field Hamiltonian in the form of $h[\rho]=T+T_{2}\left(\nu_{12} \rho_{12}\right)$. Here, $\mathrm{T}$ denotes matrix elements of kinetic part while second part corresponds to mean-field potential. $v_{12}$ is the antisymmetric two-body interaction potential, $\rho_{12}$ is the two-body density and $T r_{2}($.) is the partial trace on the degrees of freedom of the second particle.

In the mean-field approximation, the exact many-body dynamical problem of interacting particles is mapped to dynamics of independent particles moving in an average selfconsistent mean-field. Despite the fact that mean-field approach generally provides a good approximation for the average properties of one-body DOFs, it generally fails to produce quantum fluctuations of collective motion and suffers from the underestimation of dissipative effects induced by the coupling between single-particle DOFs with more complex internal DOFs. To overcome these deficiencies and improve mean-field dynamics beyond mean-field approaches are required. In this work one of the beyond mean-field approaches so called the stochastic mean-field approach is investigated.

\footnotetext{
* Corresponding author.

E-mail address: iulgen@ankara.edu.tr
} 
This study is organized as follows. In section 2, the stochastic mean-field approach is introduced and its extension to higher order moments is discussed. In section 3, the SMF approach is performed to dynamics of a schematic model which is called the Fermi-Hubbard model. Results is presented. In the end, the conclusions are given in section 4 .

\section{The Stochastic Mean-Field Approach}

In the SMF approach (Ayik 2008), the initial one-body density, $\rho(0)$, is replaced by an ensemble of stochastic initial one-body densities, $\rho^{\lambda}(0)$. Here $\lambda$ stands for event label. Each of these densities evolves with its self-consistent mean-field equation,

$i \hbar \frac{\partial \rho^{\lambda}}{\partial t}=\left[h\left[\rho^{\lambda}\right], \rho^{\lambda}\right]$

For each event, the "event" expectation value of a one-body observable $A$ is given by

$$
\langle A\rangle^{\lambda}=\operatorname{Tr}\left(\rho^{\lambda} A\right)
$$

The expectation values of an observables are obtained by ensemble averages in this approach. So, the expectation value of the one-body observable is defined as,

$\overline{\langle A\rangle^{\lambda}}=\mathrm{T} r\left(\overline{\rho^{\lambda}} A\right)=\sum_{i j} \overline{\rho_{i j}^{\lambda}} A_{j i}$

Overline states averaging in the form of

$$
\overline{\rho_{i j}^{\lambda}}=\lim _{\mathrm{N} \rightarrow \infty} \frac{1}{\mathrm{~N}} \sum_{\lambda=1}^{\mathrm{N}} \rho_{i j}^{\lambda}
$$

where $\mathcal{N}$ stands for for the number of events in the ensemble. The quantum variance of the one-body operator is defined by

$$
\begin{aligned}
\sigma_{A}^{(2)} & =\overline{\left(\langle A\rangle^{\lambda}-\overline{\langle A\rangle^{\lambda}}\right)^{2}}=\overline{\left[\operatorname{Tr}\left(\delta \rho^{\lambda} A\right)\right]^{2}}, \\
& =\sum_{i j k l} \overline{\delta \rho_{i j}^{\lambda} \delta \rho_{k l}^{\lambda}} A_{j i} A_{l k},
\end{aligned}
$$

where $\delta \rho^{\lambda}=\rho^{\lambda}-\overline{\rho^{\lambda}}$.

In the SMF approach in order to describe compatible quantal description of the complex many-body system, some restrictions on initial stochastic one body density matrices are required. Each initial stochastic matrix element, $\rho_{i j}^{\lambda}(0)$, should be specified such that initial mean and second central moment of one-body operator coincide with those obtained by using quantal one, $\rho(0)$. By using basis vectors $\{|i\rangle\}$ which diagonalize initial one-body density matrix, the mean and variance of an operator $A$ are given by

$$
\langle A(0)\rangle=\sum_{i} n_{i} A_{i i}
$$

$$
\left\langle A^{2}(0)\right\rangle-\langle A(0)\rangle^{2}=\frac{1}{2} \sum_{i j}\left[n_{i}\left(1-n_{j}\right)+n_{j}\left(1-n_{i}\right)\right] A_{j i} A_{i j},
$$

where, $n_{i}$ is the occupation number that can take values 0 or 1 initially. By considering Eqs. (6)-(9) we deduce that the ensemble description matches with quantal version if we have

$$
\overline{\rho_{i j}^{\lambda}(0)}=n_{i} \delta_{i j}
$$

and

$$
\overline{\delta \rho_{i j}^{\lambda}(0) \delta \rho_{k l}^{\lambda}(0)}=\delta_{i l} \delta_{j k} \frac{1}{2}\left[n_{i}\left(1-n_{j}\right)+n_{j}\left(1-n_{i}\right)\right] .
$$

The stochastic matrix elements, $\delta \rho_{i j}^{\lambda}(0)$, are proposed in the original work to be Gaussian random numbers satisfying Eqs. (10) and (11).

The extension of the original SMF approach is presented in (Ulgen et al. 2019). In this reference, the stochastic initial conditions are defined more accurately compared with the original SMF approach. Some additive restrictions on initial stochastic matrix elements are obtained by considering third and fourth central moments of one-body observable. Incidental to analyses of conditions Eqs. (10), (11) and additional restrictions coming from the higher order moments, it is deduced that in order to specify initial stochastic matrix elements in the ensemble, probability distribution with small kurtosis should be used (Ulgen et al. 2019). This argument is tested by simulating and comparing the SMF dynamics with three different initial distributions with each other as well as exact and MF dynamics for the modified version of the LipkinMeshkov-Glick model. It is shown that in one case (the so called two point initial distribution with smallest kurtosis) the solutions of the SMF approach are close to the exact solutions. The work is concluded with a proposal for using two-point distribution instead of Gaussian in the SMF dynamics (Ulgen et al. 2019).

\section{Application}

In this section, we apply the SMF approach with two-point distribution with kurtosis $Y_{T}=1$ as well as Gaussian with kurtosis $Y_{G}=3$, uniform with kurtosis $Y_{U}=1.8$ and bimodal with kurtosis $Y_{B}=1.4$ distributions to the Fermi-Hubbard model and show that indeed the SMF approach with two-point distribution gives a better agreement with the exact dynamics than SMF approach with other distributions.

\subsection{The Fermi-Hubbard Model}

We consider the Fermi-Hubbard model which has been extensively used as a qualitative description of some properties of solids such as "(i) the electronic properties of solids with narrow bands, (ii) band magnetism in iron, cobalt, nickel, (iii) the mott metal-insulator transition, (iv) electronic properties of high-Tc cuprates in the normal state" (Essler et al. 2005). The Hubbard model is derived from a system of

and 
electrons interacting with each other and a static lattice. The Hamiltonian of such a system is given by

$$
H=\sum_{i=1}^{N}\left(\frac{p_{i}^{2}}{2 m}+V_{e i}\left(x_{i}\right)\right)+\sum_{i<j} V_{e e}\left(\left|x_{i}-x_{j}\right|\right),
$$

where $V_{e i}\left(x_{i}\right)=-e^{2} / x_{i}$ is electron-lattice interaction which represents a periodic positive background of ions interacting with electrons. The two-body potential $V_{e e}$ is electron-electron Coulomb interaction. There are two assumptions that lead to the Hubbard model: (i) Electrons are tightly bound to their host ions and the ions are far enough not to allow hopping (tunneling) among different ions except neighboring ions (tight binding approximation). (ii) Only the electrons on the same ion can interact with each other (on site interaction). The resulting Hamiltonian reads as

$$
H=-J \sum_{\langle i, j\rangle, \sigma} c_{i, \sigma^{c}}^{\dagger} c_{j, \sigma}+U \sum_{i} n_{i, \uparrow} n_{i, \downarrow}
$$

where $\boldsymbol{c}_{\boldsymbol{i}, \boldsymbol{\sigma}}^{\dagger}$ creates an electron in the site $\boldsymbol{i}$ with a spin projection $\boldsymbol{\sigma}, \boldsymbol{n}_{\boldsymbol{i}, \boldsymbol{\sigma}}=\boldsymbol{c}_{\boldsymbol{i}, \boldsymbol{\sigma}}^{\dagger} \boldsymbol{c}_{\boldsymbol{i}, \boldsymbol{\sigma}}$, and $\langle\boldsymbol{i}, \boldsymbol{j}\rangle$ means nearest neighbors. $\boldsymbol{J}$ represents tunneling strength and $\boldsymbol{U}$ the interaction strength. In Fig. (1) a schematic illustration of the Hubbard model with 6 sites and 6 electrons (3 spin up and 3 spin down) is shown.

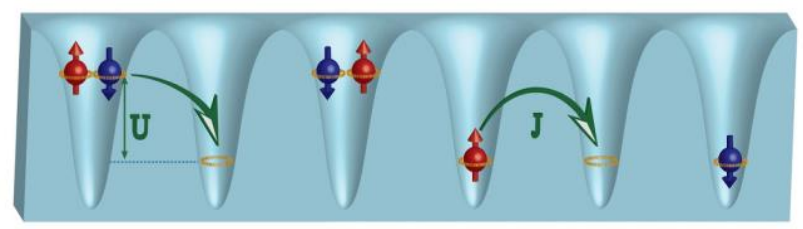

Figure 1: Schematic illustration of the Hubbard model

\subsubsection{Exact, mean-field and stochastic mean-field dynamics}

The Hubbard model is also widely used as a benchmark for numerical approaches to finite fermionic and bosonic systems. The Hubbard model is exactly solvable in one dimension by Bethe ansatz which leads to Lieb-Wu equations (Essler et al. 2005). The exact numerical solution of Hubbard model is also possible by diagonalization of the Hamiltonian or by using quantum Monte-Carlo techniques. The former technique has the drawback that the size of the matrix to be diagonalized increases very rapidly with the number of sites and the latter techniques have the drawback that the numerical errors grow rapidly, hence the results are not reliable. Even though the diagonalization technique is impossible for very large systems, still it can be used for a system of 10 half-filled sites in one dimension (Kingsley and Robinson 2013). For half-filled case with $\mathrm{N}$ sites there are

$C(2 N, N)=\frac{(2 N) !}{N ! N !}$ configurations. We consider $\boldsymbol{N}=\mathbf{8}$ sites for which there are 12870 configurations. A Hamiltonian of such size $(\mathbf{1 2 8 7 0} \times$ 12870) can be diagonalized since the matrix is sparse and there are many effective algorithms, most of which use the iterative Lanczos method, that diagonalize sparse matrices. In this study, we will follow the technique proposed in Ref. (Lin 1990) and used in Refs. (Kingsley and Robinson 2013, Lin and Gubernatis 1993, Jafari 2008 and Siro and Harju 2012). For the actual computation, the matrix size is reduced by using the conservation of the z component of the total spin $\boldsymbol{S}_{\boldsymbol{z}}$ since it commutes with the Hamiltonian. It is given by $S_{Z}=\frac{1}{2}\left(N_{\uparrow}-N_{\downarrow}\right)$

where $\boldsymbol{N}_{\boldsymbol{\sigma}}=\sum_{\boldsymbol{i}} \boldsymbol{n}_{\boldsymbol{i}, \boldsymbol{\sigma}}$. Specifically, we consider an initial state that satisfies $\boldsymbol{S}_{\boldsymbol{z}}\left|\boldsymbol{\psi}_{\mathbf{0}}\right\rangle=\mathbf{0}$ which is conserved during the dynamical evolution (Obviously $\mathrm{N}$ must be even.). Then, the number of configurations is

$C(N, N / 2)^{2}=\left[\frac{N !}{(N / 2) !(N / 2) !}\right]^{2}$.

For $N=8$, we get 4900 configurations. The exact dynamics is obtained by diagonalizing the corresponding Hamiltonian.

We consider the initial state with all particles filling the left sites, that is 4 spin up and 4 spin down fermions on the 4 left sites with the remaining 4 sites on the right empty. This initial state has spin symmetry and since Hamiltonian Eq. (13) preserves the spin symmetry, the exact one-body density preserves the spin symmetry as well. Hence, the one-body density preserves the property

$\rho_{i, \sigma^{\prime} ; j, \sigma}=\rho_{i, \sigma, j, \sigma} \delta_{\sigma^{\prime}, \sigma}$

at all times, where $\rho_{i, \sigma ; ; j, \sigma}=\left\langle c_{j, \sigma}^{\dagger} c_{i, \sigma \prime}\right\rangle$. Then, by using the shorthand notation $\rho_{i, j, \sigma} \equiv \rho_{i, \sigma ; j, \sigma}$, the mean-field equation of the Hubbard model can be written as

$$
\begin{aligned}
i \hbar \frac{d}{d t} \rho_{i, j, \sigma}= & -J\left(\rho_{i+1, j, \sigma}-\rho_{i, j-1, \sigma}+\rho_{i-1, j, \sigma}-\rho_{i, j-1, \sigma}\right) \\
& +U\left(\rho_{i, i, \sigma}-\rho_{j, j, \sigma}\right) \rho_{i, j, \sigma} .
\end{aligned}
$$

The SMF equations of motion are directly obtained from the last equation by replacing all one-body densities with the stochastic one, $\rho \rightarrow \rho^{\lambda}$. The original SMF approach has been applied to the Hubbard model (Lacroix et al. 2014).

\subsubsection{Results}

Figure 2 shows the time evolution of the expectation value of the center of mass position of the fermions defined as

$$
\left\langle x_{c m}\right\rangle=\sum_{i=1}^{N} \frac{x_{i} n_{i}(t)}{N}=\sum_{i=1}^{N}\left(i-\frac{1}{2}\right) \frac{n_{i}(t)}{N},
$$

for different coupling strengths. Here, the sites are considered to be on the positions $x_{i}=\left(i-\frac{1}{2}\right)$ for $i=1,2, \ldots, N$. For simplicity site positions are chosen dimensionless. First subfigure in Figure 2 shows that MF evolutions starts the 
deviate from exact solution at around $t=4.0 \mathrm{~J}^{-1}$ when SMF approach gives better approximation at early times of the evolution in strong coupling regime, $\mathrm{U}=1 \mathrm{~J}$. According to second subfigure in Fig. 2 departure of the MF solution occurs at $t=40 \mathrm{~J}^{-1}$. All the SMF solutions are better than MF solutions at all times. Comparing all the SMF dynamics with the exact one in this subfigure, we see that SMF approach with twopoint distribution, SMF (T), presents better estimation than SMF with rest of the distributions. As shown at third subfigure in Fig. 2 that difference between the SMF and exact evolutions occurs at $t=500.0 \mathrm{~J}^{-1}$ in weak coupling limit, $\mathrm{U}=0.01 \mathrm{~J}$ meanwhile MF gives worse solution when time increases. As seen in Fig.2 with long time evolution that although there is a big discrepancy between the oscillation amplitudes of the MF solution and the exact one, there is good accordance between the exact and SMF dynamics, especially SMF (T). Hence we deduce that the SMF approach with two-point distribution, SMF (T), estimates the best dissipation effects.
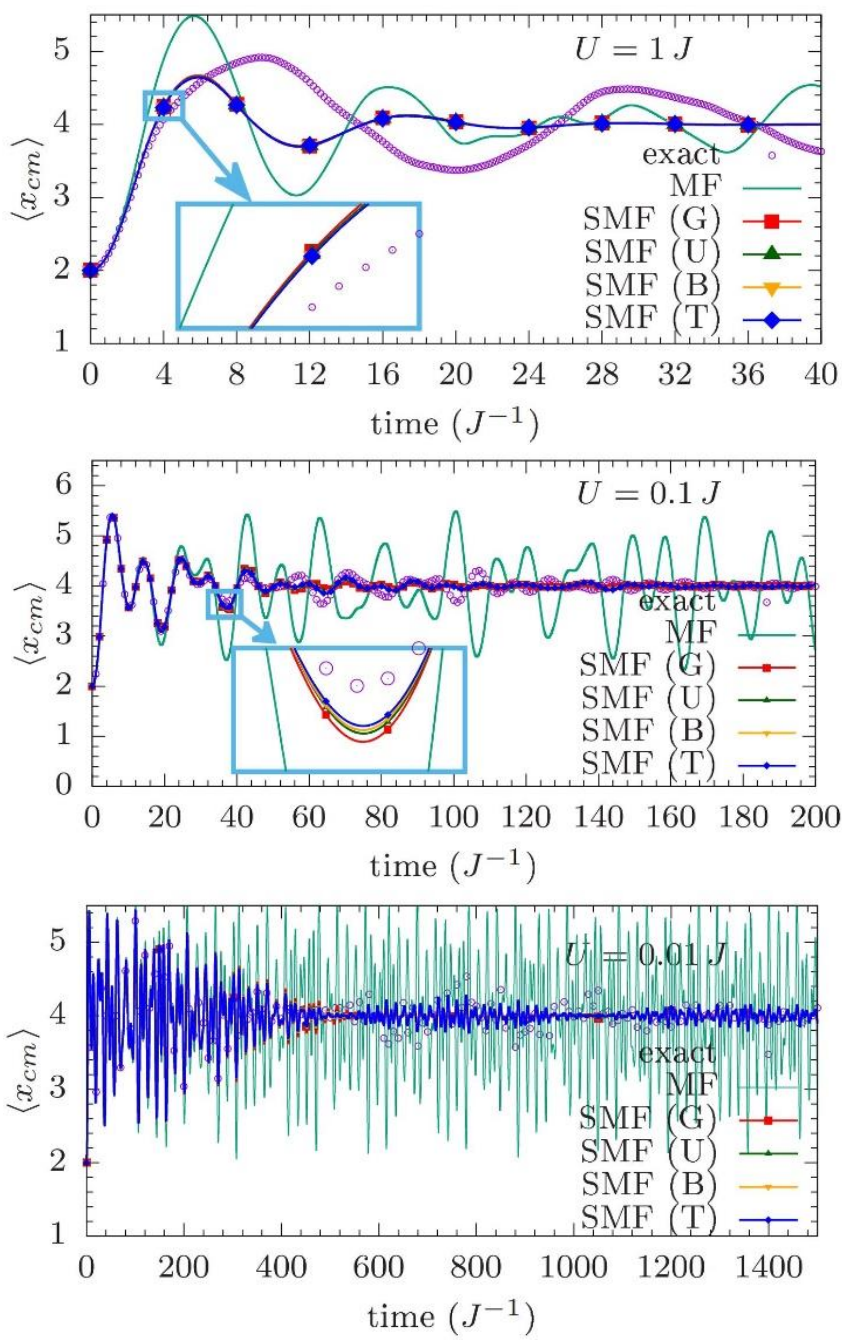

Figure 2: The center of mass position, given by Eq.(19), is plotted versus time for different coupling strenghts, $U=1 \mathrm{~J}(\mathrm{a}), U=0.1 \mathrm{~J}(\mathrm{~b})$, and $U=0.01 \mathrm{~J}(\mathrm{c})$. The exact solution is shown with the circle. The SMF solutions with Gaussian (G), uniform (U), bimodal (B) and two-point (T) distributions are indicated by the red line with boxes, the green line with triangles, the yellow line with reversed-triangles and the blue line with diamonds, respectively.

Similar behaviour can be seen from Figure 3 which shows time evolution of one-body entropy defined as

$S=-\operatorname{Tr}[\rho \ln \rho+(1-\rho) \ln (1-\rho)]$,

where $\rho$ is the one-body density operator. It indicates how much the state of the system departures from independent particle picture and measures thermalization. In the SMF approach, the one-body entropy is calculated by
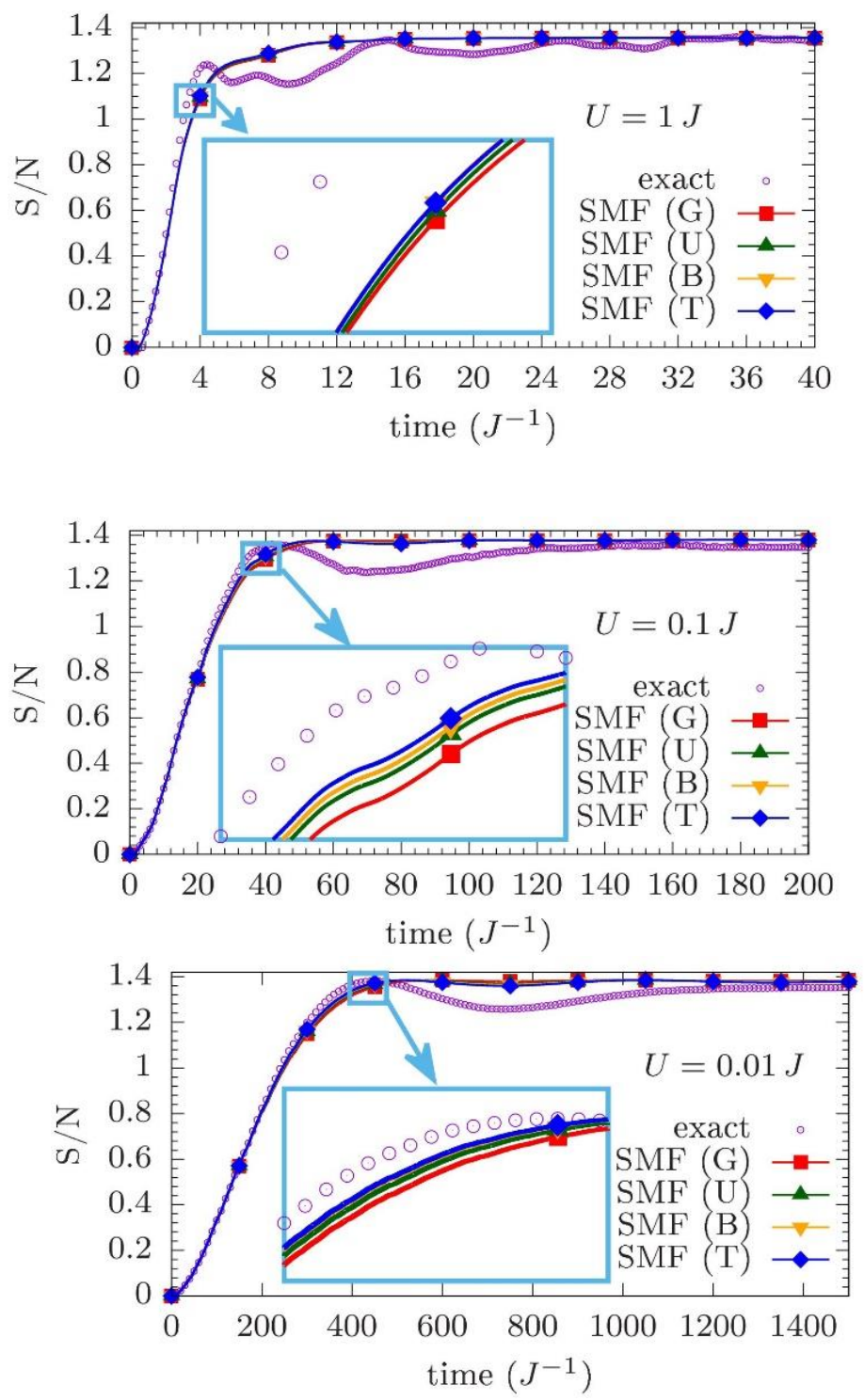

Figure 3: The one-body entropy per particle, given by Eq.(21), is plotted versus time for different coupling strenghts, $U=1 J(\mathrm{a}), U=$ $0.1 \mathrm{~J}(\mathrm{~b})$, and $U=0.01 \mathrm{~J}(\mathrm{c})$. The exact solution is shown with the black line. The SMF solutions with Gaussian (G), uniform (U), bimodal (B) and two-point $(\mathrm{T})$ distributions are indicated by the red line with boxes, the green line with triangles, the yellow line with reversedtriangles and the blue line with diamonds, respectively. 
$S=-\operatorname{Tr}\left[\overline{\rho^{\lambda}} \overline{\ln \rho^{\lambda}}+\left(1-\overline{\rho^{\lambda}}\right) \ln \left(1-\overline{\rho^{\lambda}}\right)\right]$.

For all the coupling strengths, the SMF solution with the twopoint distribution is a better approximation to the exact one as seen in the inset figures. The validity time of the SMF solutions show the the property $\Delta t_{\mathrm{val}} \propto U^{-1}$ (Polkovnikov 2003). The differences between the results for the four distributions are small which suggests that the effects of different distributions can be small or large depending on the physical case as well as the coupling strength. The reason of dependence on coupling strength is already well known however dependence of the physical systems might be related to the effect of the missing couplings that the classical distributions are not able to take into account. These missing couplings can be more important for some and less important for other quantum systems.

\section{Conclusions}

Mean-field (MF) approach is widely used in many areas in physics. Although it provides a good description to determine properties of interacting many-body complex systems, it suffers from some missing effects. Absence of quantal correlations is the major issue which causes the MF approach to lose validity in long time evolution. Besides, another drawback is the underestimation of the dissipation effect which requires to be incorporated to dynamics. For the purpose of improving predicting power of the approximation, beyond mean-field approaches are needed. One of them is so called the stochastic mean-field (SMF) approach.

The SMF approach upgrades mean-field approach by taking into account initial quantum fluctuations. In the SMF approach stochastic initial conditions are introduced on top of the meanfield in such a way that the initial state of system is replaced by an ensemble of stochastic initial one-body densities. Mean value of a one-body operator is computed by taking statistical ensemble average. In the original SMF approach, initial stochastic one-body densities are specified in such a way that quantum mean and variance of a one-body observable match with values obtained by statistical ensemble average. By doing this specification, usage of Gaussian distribution in the stochastic matrix elements is assumed in the original work (Ayik 2008). In the work of Ulgen et al. (2019), by considering higher order moments it is investigated that exploiting probability distribution with minimum kurtosis for the initial stochastic one-body density matrix elements gives the best approximation instead of using Gaussian distribution.

In this work, we apply the SMF approach with four different initial distributions for the Fermi-Hubbard model. The exact solution is compared with the solutions of the MF equations and SMF equations. Four versions of initial distributions denoted Gaussian with kurtosis $Y_{G}=3$, uniform with kurtosis $Y_{U}=1.8$, bimodal with kurtosis $Y_{B}=1.4$ and two-point with kurtosis $Y_{T}=1$ are used. It is shown that the SMF approach with two-point distribution is the best approximation in the weak coupling regime as well as strong coupling strengths. However, for the Fermi-Hubbard model the difference between different SMF evolutions is very small while it is more pronounced for the model in Ref. (Ulgen et al. 2019).

\section{Acknowledgements}

We gratefully acknowledge useful discussions with Denis Lacroix. This work was supported in part by the Scientific and Technological Research Council of Turkey (TUBITAK).

\section{References}

Ayik, S., 2008. A stochastic mean-field approach for nuclear dynamics. Phys. Lett. B 658, 174.

Ayik, S., Yilmaz, B., Yilmaz O., and Umar, A. S., 2019. Quantal diffusion approach for multinucleon transfers in $\mathrm{Xe}+\mathrm{Pb}$ collisions, Phys. Rev. C 100, 014609.

Bogoliubov, N. N., 1946. Kinetic Equations, J. Phys. (URSS) 10, 256.

Born, H. and Green, H.S., 1946. A general kinetic theory of liquids I. The molecular distribution functions. Proc. R. Soc. A 188, 10.

Essler, F. H. L. Frahm, H., Göhmann, F., Klümper, A. and Korepin, E., 2005. The One-Dimensional Hubbard Model. Cambridge University Press.

Jafari, S. A., 2008. Introduction to Hubbard model and exact diagonalization. IJPR 8, 113.

Kingsley, O. N. and Robinson, 0., 2013. Exact Diagonalization of the Hubbard Model: Ten-electrons on Ten-sites. Res. J. Appl. Sci. Eng. Technol., 6(21), 4098.

Lin, H. Q., 1990. Exact diagonalization of quantum-spin models. Phys. Rev. B 42, 6561.

Lin, H. Q. and Gubernatis J. E., 1993. Exact diagonalization methods for quantum systems. Computers in Physics, 7, 400 .

Kirwood, J.G., 1946. The statistical mechanical theory of transport processes I. General theory. J. Chem. Phys. 14, 180

Lacroix, D., Hermanns, S., Hinz, C. M. and Bonitz, M., 2014. Ultrafast dynamics of finite Hubbard clusters: A stochastic mean-field approach. Phys. Rev. B 90, 125112.

Lacroix, D. and Ayik, S., 2014. Stochastic quantum dynamics beyond mean field, Eur. Phys. J. A 50: (94).

Polkovnikov, A., 2003. Quantum corrections to the dynamics of interacting bosons: Beyond the truncated Wigner approximation. Phys. Rev. A 68, 053604.

Siro, T. and Harju, A., 2012. Exact diagonalization of the Hubbard model on graphics processing units. Comp. Phys. Comm. 183, 1884.

Ulgen I., Yilmaz, B., Lacroix, D., 2019. Impact of the initial fluctuations on the dissipative dynamics of interacting Fermi systems: a model case study, arXiv:1908.05520v1.

Yilmaz, B., Ayik, S., Yilmaz O., and Umar, A. S., 2018. Multinucleon transfer in $58 \mathrm{Ni}+60 \mathrm{Ni}$ and $60 \mathrm{Ni}+60 \mathrm{Ni}$ in a stochastic mean-field approach, Phys. Rev. C 98, 034604. 